\title{
Passively Enhanced Natural Convection Heat Transfer via Swirl Effect
}

\author{
Luke Di Liddo, David Naylor \\ Dept. of Mechanical and Industrial Engineering, Ryerson University \\ Toronto, Canada \\ ldiliddo@ryerson.ca,dnaylor@ryerson.ca
}

\begin{abstract}
A numerical and experimental study of the free convective heat transfer rate from a heated circular disk experiencing swirling flow has been carried out using ANSYS Fluent and a Mach Zehnder Interferometer (MZI). A horizontal, flat, isothermal disk has been subjected to radially swirling flow by the placement of stationary angled blades, or vanes, around the circumference of the disk. An examination of the flow pattern on a plane approximately normal to the primary flow revealed regions of downwash and upwash near the surface of the disk. The vortices in the secondary flow generated areas of increased and decreased surface heat flux corresponding to the regions of downwash and upwash, respectively. The RNG kepsilon turbulence model was used to obtain the disk's overall Nusselt number for $1.28 \times 10^{6} \leq R a_{D} \leq 2.56 \times 10^{8}$. The numerical model was used to compute and compare Nusselt numbers between vane designs of varying height, angle, length, thickness, and number. Preliminary vane design recommendations are made. Results show passive natural convection heat transfer enhancement of up to $35 \%$ via the swirl effect when compared to the model with no swirl.
\end{abstract}

Keywords: natural convection, disk, swirling flow, MZI, heat transfer enhancement, $C F D$

\section{INTRODUCTION}

Performance and power consumption in electronics design are coupled phenomena. As semiconductor technology advances, the power consumption of computer chips has increased and consequently, the requirements for heat dissipation have increased. The heat dissipation rate is often the limiting factor in circuit board performance and is a factor in reducing the lifespan of light emitting diodes (LEDs) [1]. As the power density of electronic devices increase, effective thermal management of these components becomes critically important to their stable operation and longevity.

Natural convection offers not only a simple cooling method, but one devoid of external power inputs or moving parts, i.e. it is a passive thermal management method. Many heat sinks have been designed on the principle of natural convection, but despite the number and effectiveness of the various designs, none consider the effect of a passively induced swirling flow on the free convective heat transfer rate.

A review of natural convection heat and mass transfer over horizontal heated surfaces is given by Corcione [2]. Correlations from experimental, numerical, and theoretical approaches cover a wide range of Rayleigh numbers for different surface geometries and fluids. The experiments of AlArabi and El-Reidy [3], Yousef et al. [4], and Kitamura and Kimura [5] are the among most relevant. They provide experimental relations between the average Nusselt number and Rayleigh number for horizontal, isothermal, heated plates under natural convection in air. Yousef et al. [4] studied the natural convection heat transfer from a horizontal square plate using a Mach Zehnder Interferometer (MZI). They reported significant temporal unsteadiness in both the average and local Nusselt numbers, with fluctuations in the average Nusselt ranging from $+23 \%$ to $-15 \%$. Kitamura and Kimura [5], in 2008, studied heat transfer from circular disks by a heat balance. They also performed visualization experiments for air and water and show the turbulent branching structure of the flow over the disk. By showing this turbulence, they correct the previous misinterpretation of the point of onset of turbulence. Prior to this study, researchers such as [3] have claimed the onset of turbulence for horizontal heated surfaces under natural convection is about $R a=4 \times 10^{7}$. However, Kitamura and Kimura visually show turbulent branching as early as $R a=$ $1 \times 10^{6}$. The reason for this discrepancy is that the turbulence initially occurs at the center of the geometry where the heat flux is lowest and as such, the effect of turbulence on heat transfer cannot be detected with non-optical methods until $R a_{D} \approx 4 \times 10^{7}$.

The present study investigates the effect of a passively induced swirling flow on the heat transfer rate from a heated circular disk. The main utility of these results is to establish whether a swirling flow can increase the disk's natural convective heat transfer rate relative to a model under no imposed swirl.

\section{PROBLEM DESCRIPTION}

This study concerns natural convection heat transfer over a horizontal, flat, isothermal, heated, upward-facing, circular disk 
at temperature $T_{s}$. The surrounding air is at ambient temperature, $T_{\infty}$. The geometry was subjected to a swirling flow induced through stationary, adiabatic, angled vanes, as illustrated in Fig. 1. A disk under no imposed swirl (no vanes) was used as a comparison to determine whether a passively induced swirling flow can increase the disk's surface heat transfer rate. A numerical model of "swirl" and "no swirl" and an experimental model of "swirl" and "no swirl" were created in this study. The numerical study was carried out using the commercial Computational Fluid Dynamics (CFD) software ANSYS FLUENT. The experimental study was carried out using an MZI. Multiple vane designs were used in the numerical model of swirl while only one vane design was studied experimentally.

The disk under imposed swirl used stationary angled vanes placed periodically around the circumference of the disk to generate swirl. The angled vanes direct incoming flow such that it approaches the circumference almost tangentially, thereby swirling the flow as it proceeds over the surface of the disk. The geometry of the no swirl model is the same as shown in Fig. 1 except without any vanes. In both models, the top face of the isothermal disk was coplanar with the surrounding insulation.

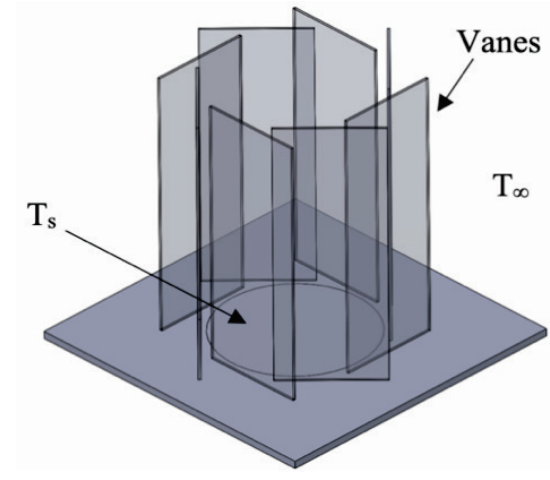

Fig. 1: Isometric view of the model with vanes.

Fig. 2 shows the location and geometry of the vanes, as defined by their number $\left(N_{v}\right)$, height $\left(H_{v}\right)$, length $\left(L_{v}\right)$, thickness $\left(t_{v}\right)$, radius from the center of the disk $\left(R_{v}\right)$, and angle from their radial line $\left(\theta_{v}\right)$. The gap length between adjacent vanes, $G_{v}$, was varied as a function of vane length, radius and angle, where $G_{v}$ is defined as the shortest distance between adjacent vanes. A parametric study was performed numerically to evaluate the effect of each of these variables on the surface heat transfer rate.

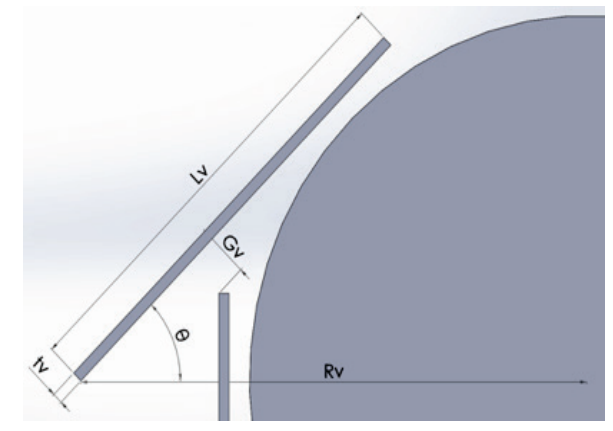

Fig. 2: A plan view of the geometric definition of the vanes.

\section{NUMERICAL PROCEDURE}

\section{A. General Numerical Procedure}

The commercial CFD software ANSYS FLUENT was used to solve this problem. A three-dimensional finite volume model was developed in FLUENT to solve for the natural convective heat transfer rate from the heated disk. Radiation heat transfer is neglected. For all fluid zones, the conservation of mass, momentum and energy were solved, as well as the transport equations for turbulent kinetic energy and turbulent kinetic energy dissipation rate. The RNG $k$ - $\varepsilon$ turbulence model was used for the swirl and no swirl models. At convergence, the residuals of the governing equations were all less than $10^{-4}$. In addition, the heat transfer rate from the disk was monitored for convergence to confirm the stability of the solution. Enhanced wall treatment, full buoyancy effects and thermal effects were used with the RNG model. The option for swirl dominated flow was included in the models with swirl. The turbulence model was used starting from $R a_{D}=1 \times 10^{6}$ because that is when turbulence first occurs at the center of the disk as confirmed visually by Kitamura and Kimura [5]. The thermophysical properties were considered constant with the exception of the air density, which was treated using the Bousinessq approximation. The air properties were evaluated at standard atmospheric pressure and at the film temperature between the surface of the heated disk and the ambient air, $T_{f}=\left(T_{s}+T_{\infty}\right) / 2$.

\section{B. Computational Domain, Boundary Conditions, and Discretization}

The computational domain was a cube whose vertical axis coincided with the central axis of the circular disk. The domain had a size of $L / R=3.5$, where $L$ is any of the cubic dimensions and $R$ is the radius of the disk. A study determined that the placement of the far field boundaries beyond the prescribed distances had no significant effect on the average heat transfer rate from the disk for both swirl and no swirl models.

The sides of the computational domain had pressure inlet boundary conditions and the top had a pressure outlet boundary condition. On the bottom face, the disk had an isothermal boundary condition and the surrounding insulation had an adiabatic insulation. For the simulations studying swirl, the vanes had an adiabatic boundary condition. The disk, insulation, and vanes were considered no-slip surfaces with no surface roughness.

An unstructured tetrahedral mesh was used to discretize the domain. The computational domain was discretized into 4 sections, as shown in Fig. 3: A, a fine mesh very close to the surface of the disk, B, a moderately sized mesh near the disk, C, a coarse mesh slightly further from the disk, and D, the coarsest mesh far away from the disk. For the simulations with vanes, a fifth section was added: E, a fine mesh near the surface of the vanes. The total number of cells was approximately $4 \times 10^{6}$, with approximately 10 times more cells in Section A than Section D. A study determined that a finer mesh did not have a significant influence the heat transfer rate from the disk. 


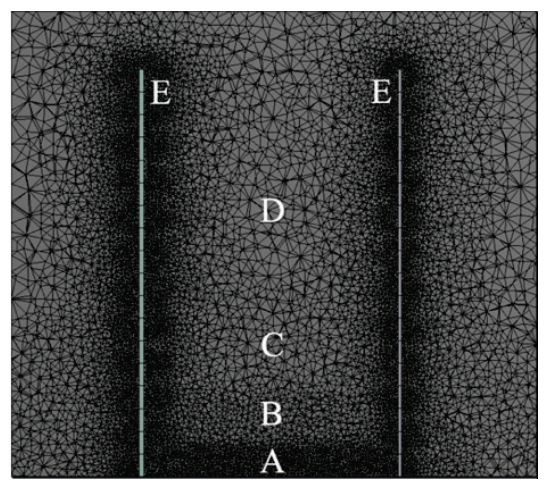

Fig. 3: Discretization scheme.

\section{Validation of Numerical Model}

In the present study, the heat transfer rates numerically computed for the no swirl model were much lower than the correlations proposed in the literature. In the laminar and transition range $\left(R a_{D}<4 \times 10^{7}\right)$, the average Nusselt number in the present study is some $60 \%$ lower than the correlations from Al-Arabi and El-Reidy [3] and Kitamura and Kimura [5]. They are also approximately $30-45 \%$ lower than the results of Yousef et al. [4], depending on the Rayleigh number. In the turbulent range $\left(R a_{D}>4 \times 10^{7}\right)$, the present correlations are approximately $5-30 \%$ lower than the published correlations, with increasing agreement as Rayleigh number increases. The largest discrepancy between the present and published correlations occurs in the laminar to turbulent transition range $\left(1 \times 10^{6}<R a_{D}\right.$ $<4 \times 10^{7}$ ), which suggests there may be a deficiency in the numerical modelling of this phenomena. No explanation can presently be given for the discrepancy between the present and published correlations and further investigation is needed. Different turbulence models such as the standard $k-\varepsilon$ and $k-\omega$ models were tried to no avail, and as noted above, a finer mesh did not significantly influence the results. Despite this discrepancy, the numerical model has approximately captured the overall physics of the flow. In Fig. 4, the numerical temperature profile in a center plane is shown, which agrees with the interferometry experiments of Yousef et al. [4]. Fig. 4 also shows good agreement with the visualization experiments of Kitamura and Kimura [5], including the turbulent branching which occurs near the center of the disk.

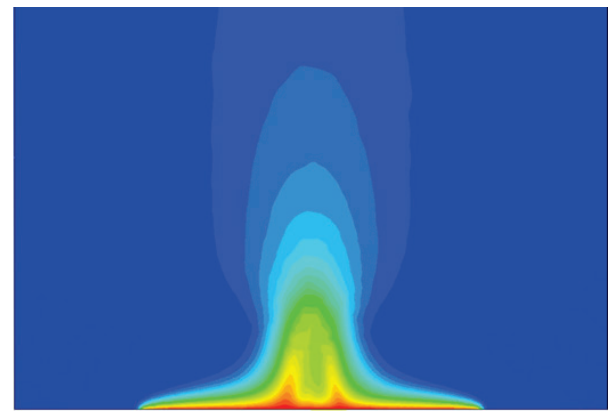

Fig. 4: Numerical temperature profile in a center plane for the model with no swirl, which agrees with the temperature profile and general flow pattern of [4] and [5]. $R a_{D}=1.3 \times 10^{7}$.

\section{EXPERIMENTAL PROCEDURE}

\section{A. Experimental Model}

An experimental model was created for both the case with swirl and without swirl for measurements in an MZI. The experimental model consisted of a circular aluminum disk, an electric heater, insulation, and vanes inserted into the insulation. The aluminum disk had a thickness to diameter ratio of 0.05 and was machined and polished flat on the top face. A sheet of insulation, with the top surface coplanar with the top face of the disk, was placed around the disk. The disk was heated from the bottom by an electric heater which itself rested on insulation. Two type-K thermocouples, at the center and near the edge of the disk, both $2 \mathrm{~mm}$ from the top surface, were used to measure the surface temperature of the disk and ensure its uniformity. The difference between these measurements was less than $0.5 \%$ of the surface temperature. The disk was heated to a surface temperature of $60^{\circ} \mathrm{C}$ in order to create a temperature difference of approximately $40^{\circ} \mathrm{C}$ from the ambient. The combination of a $20 \mathrm{~cm}$ optical path length (the diameter of the disk) and a $40^{\circ} \mathrm{C}$ temperature difference produced enough fringes for an accurate measurement while keeping diffraction error near the surface within a reasonable bound [6]. The experimental setup was identical for the swirl and no swirl models except in the swirl model, pieces of cardboard were inserted into the surrounding insulation to act as the vanes.

\section{B. Interferometric Procedure}

Laser interferometry is an optical method that uses the wave nature of light to measure the changes in refractive index of a heated medium. The changes in refractive index can then be related to the temperature field in the heated medium, allowing measurement of the heat transfer rate and visualization of the real time temperature field. The optical equipment was mounted on a $360 \mathrm{~kg}$ vibration-isolated bench located in a large unventilated enclosure. The system uses a $15 \mathrm{~mW}$ He-Ne laser as a light source. The laser was equipped with a spatial filter to reduce high frequency optical noise and uses a set of input optics to expand the beam to a $20 \mathrm{~cm}$ diameter collimated beam. Additional details regarding the theory and operation of the MZI are given by Naylor [7,8] and Goldstein [9].

In the current experiment, the vanes block the light in the test beam. For this reason, only limited optical measurements were possible. Two rectangular holes $(15 \mathrm{~mm}$ by $20 \mathrm{~mm})$ were made in opposing vanes to allow the transmission of a small portion of the test beam adjacent to the surface of the disk. These two holes allowed the measurement of the beam-averaged temperature field across the centre of the disk. As described below, the beam-averaged temperature field was used to measure average convective heat flux along a line that passes from edge to edge, across the centre of the disk. Since it was not possible to measure the heat flux optically over the entire disk, the average heat flux along a line that passes through the disk's centre $\left(\bar{q}_{c e n}\right)$ was used as a proxy for the total convective heat transfer rate. Changes in this averaged heat flux along a single line were used to provide a quantitative indication of the effect 
of the vanes on the convective heat transfer rate from the entire plate. The implications and limitations of this approach will be discussed further in this section.

The interferometer integrates changes in the refractive index along the test beam (z-direction). Neglecting refraction effects, in infinite fringe mode the fringe shift order $(\varepsilon)$ is related to the difference in refractive index as follows [8]:

$$
\varepsilon=\frac{1}{\lambda_{0}} \int_{0}^{L}\left(n_{r e f}-n(x, z)\right) d z
$$

where $\mathrm{n}$ is the refractive index of the air and $\lambda_{0}$ is the wavelength of the laser light. For a He-Ne laser, $\lambda_{0}=632.8 \mathrm{~nm}$. In the present experiment, the optical path length is approximately equal to the diameter of the disk $(L \approx D)$. The subscript "ref" refers to the reference (ambient) conditions.

The Gladstone-Dale equation is used to relate the refractive index of the air to its density. The ideal gas equation of state relates the density $(\rho)$ to the temperature of the air. With these substitutions, (1) becomes [8]:

$\varepsilon=\frac{G}{\lambda_{0}} \int_{0}^{L}\left(\rho_{\text {ref }}-\rho\right) d z=\frac{G p}{\lambda_{0} R} \int_{0}^{L}\left(\frac{1}{T_{\text {ref }}}-\frac{1}{T(x, z)}\right) d z$

where $G$ is the Gladstone-Dale constant in air for a He-Ne laser and is equal to $0.225 \times 10^{-3} \mathrm{~m}^{3} / \mathrm{kg}, R$ is the ideal gas constant for air, $T$ is absolute temperature, and $p$ is the absolute air pressure. In this experiment, atmospheric pressure was measured using a mercury barometer. Differentiating (2) with respect to $x$, recognizing $T_{r e f}$ is a constant and evaluating the result at the surface (i.e., at $x=0$ where $T=T_{s}$ ) yields:

$$
\left.\frac{\partial \varepsilon}{\partial x}\right|_{x=0}=\left.\frac{-G p}{\lambda_{0} R T_{s}^{2}} \int_{0}^{L} \frac{\partial T}{\partial x}\right|_{x=0} d z=\left.\frac{-G p L}{\lambda_{0} R T_{s}^{2}} \frac{\overline{\partial T}}{\partial x}\right|_{x=0}
$$

In (3), the beam-averaged surface temperature gradient is:

$$
\left.\overline{\frac{\partial T}{\partial x}}\right|_{x=0}=\left.\frac{1}{L} \int_{0}^{L} \frac{\partial T}{\partial x}\right|_{x=0} d z
$$

Noting that the heat transfer at the surface occurs by pure conduction, (3) can be rearranged to give the beam-averaged surface heat flux along the disk's centre $\left(\bar{q}_{c e n}\right)$ in terms of the fringe gradient at the surface:

$$
\bar{q}_{c e n}=-\left.k_{s} \frac{\overline{\partial T}}{\partial x}\right|_{x=0}=\left.\frac{k_{s} \lambda_{0} R T_{s}^{2}}{G p L} \frac{\partial \varepsilon}{\partial x}\right|_{x=0}
$$

The fringe shift gradient at the surface was measured experimentally by analyzing the interferograms. A custom MATLAB digital image processing algorithm based on the nonlinear regression method of Slepicka and Cha [10] was used to measure the fringe gradient near the surface. To reduce the effects of optical noise, the surface fringe gradient was measured at ten equal intervals within $1 \mathrm{~mm}$ of the disk's centerline. The average of these ten scans was used in (5) to calculate the beam-averaged heat flux. Further details on the image analysis procedure can be found in [11].
Due to significant unsteadiness in the temperature field, where temporal fluctuations in $\overline{N u}_{D}$ from photo to photo ranged from $+28 \%$ to $-16 \%$, the average from fifteen photographs was used to calculate $\overline{N u}_{D}$ for each Rayleigh number. Ten to fifteen photographs were sufficient to obtain a stable average. The magnitude of these fluctuations approximately agrees with those observed in an MZI experiment by Yousef et al. [4].

The present interferometry experiment was performed at $R a_{D}=2.41 \times 10^{7}$. For the experiment with no swirl, the measured average heat transfer across the centre of the disk $\left(\bar{q}_{c e n}\right)$ was 25$35 \%$ lower than predicted by published correlations for the average Nusselt number. A lower heat flux measurement is expected because of the nature of the current measurement. As discussed above, the line-averaged heat flux across the centre of the disk was measured, rather than the area-averaged heat flux for the entire disk. For a circular disk, the region of highest surface heat flux is near the outer edge, which corresponds to a large annular area. The lowest surface heat flux occurs toward the disk's center, which corresponds to a comparatively small area. Hence, the beam-averaged heat flux across the centre of the disk is expected to be lower than the area-averaged heat flux for the entire disk. Nevertheless, within limitations of interferometry for this geometry, the measurement of $\bar{q}_{c e n}$ can be expected to give a reliable indication of heat transfer enhancement produced by a swirling flow.

\section{RESUlTS AND DisCUSSION}

In the numerical and experimental models, the average Nusselt number, $\overline{N u}_{D}$, over the top surface of the disk was calculated for a given Rayleigh number $R a_{D}$. In the numerical model, $\overline{N u}_{D}$ was found for $1.3 \times 10^{6}<R a_{D}<2.5 \times 10^{8}$, where:

$$
\begin{gathered}
R a_{D}=G r_{D} \cdot \operatorname{Pr}=\frac{g \beta\left(T_{s}-T_{\infty}\right) D^{3} \rho_{f}^{2} c_{p_{f}}}{\mu_{f} k_{f}} \\
\overline{N u_{D}}=\frac{\bar{q} D}{\left(T_{s}-T_{\infty}\right) k_{s}}=\frac{\bar{h} D}{k_{s}}
\end{gathered}
$$

In (6), $\beta$ is the coefficient of volumetric expansion, which was calculated as $1 / T_{f}$, where $T_{f}$ is the absolute film temperature. The conductivity $k$, constant pressure specific heat $c_{p}$, and dynamic viscosity $\mu$ of air were obtained from the property data of Touloukian et al. [12], Touloukian and Makita [13], and Touloukian et al. [14], respectively. The subscript "f" indicates the value was taken at the film temperature and the subscript "s" indicates the value was taken at the surface temperature of the disk. The density $\rho$ of air was calculated using the ideal gas equation of state.

As previously noted, the present numerical and experimental results are lower than those from published literature. For this reason, the relative difference between the swirl and no swirl models will become the focus of this study. The relative values of heat transfer between the swirl and no swirl models are expected to be meaningful, since both numerical results were generated with the same turbulence model. The experimental 
measurements of the swirl and no swirl cases used the same beam-averaging approach across the centre of the disk.

\section{A. Heat Transfer Comparison between Swirl and No Swirl Models}

Four different vane designs, called Vanes 1, 2, 3 and 4, were tested numerically to see which design could give the highest increase in heat transfer compared to the model with no swirl. Table 1 lists the parameters for each vane design while Fig. 2 shows the geometric definition of the design parameters.

Table 1: Vane design parameters nondimensionalized with the radius $R$ of the circular disk.

\begin{tabular}{|c|c|c|c|c|c|c|c|}
\hline Design & $N_{v}$ & $H_{v} / R$ & $t_{v} / R$ & $R_{v} / R$ & $L_{v} / R$ & $\theta_{v} / R$ & $G_{v} / R$ \\
\hline Vanes 1 & 8 & 3.5 & 0.03 & 1.5 & 1.2 & $45^{\circ}$ & 0.19 \\
\hline Vanes 2 & 8 & 3.5 & 0.03 & 1.5 & 1 & $45^{\circ}$ & 0.33 \\
\hline Vanes 3 & 8 & 3.5 & 0.03 & 1.5 & 1.3 & $45^{\circ}$ & 0.12 \\
\hline Vanes 4 & 8 & 3.5 & 0.03 & 1.6 & 1.2 & $45^{\circ}$ & 0.26 \\
\hline
\end{tabular}

The numerical model showed a significant increase in heat transfer for the model with swirl compared to the model with no swirl. Fig. 5 shows the ratio of average Nusselt numbers between the model with swirl and with no swirl, $\frac{\overline{N u}_{D, \text { swirl }}}{\overline{N u}_{D, \text { no swirl }}}$, for different vane designs. Fig. 5 shows this ratio reaches a maximum of approximately 1.38 for the design called "Vanes 3 " at $R a_{D} \approx 1.3 \times 10^{7}$. This is a significant increase for a passively induced mechanism where no external power input or moving parts were used. Between the iterations, one can see that a smaller gap length tends to increase the heat transfer rate from the disk. However, a smaller gap length will eventually choke the flow and decrease heat transfer as $G_{v}$ approaches 0 .

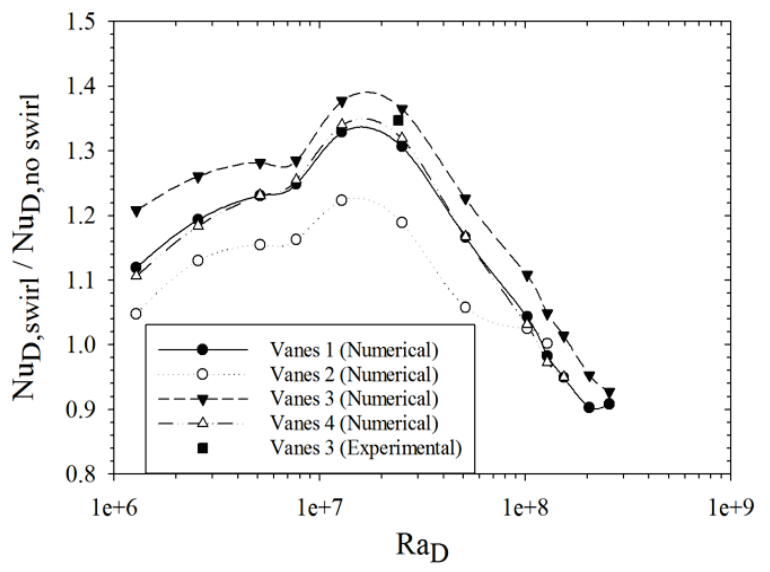

Fig. 5: Numerically and experimentally measured increase in heat transfer between the swirl and no swirl models for different vane designs.

Fig. 5 also shows good agreement between the experimentally and numerically predicted increase in the heat transfer ratio. At $R a_{D} \approx 2.4 \times 10^{7}$, the value of $\frac{\overline{N u}_{D, \text { swirl }}}{\overline{N u}_{D, \text { no swirl }}}$ is 1.37 for the numerical model of the "Vanes 3" design and 1.35 for the experimental model of the same configuration. The agreement between the numerical and experimental models suggests that a heat transfer increase of up to approximately $35 \%$ may be achieved at certain Rayleigh numbers. However, the numerical models also show that a slight decrease in heat transfer is possible at higher Rayleigh numbers $\left(R a_{D} \approx 1 \times 10^{8}\right)$, potentially when the vanes begin to choke the incoming flow. Further work is needed to expand and validate this data across a wider range of Rayleigh numbers and different vane designs.

The numerical results also show that the stationary vanes were able to generate a strong swirling flow over the surface of the disk. Fig. 6 shows streamlines of the swirling flow on a plane a distance of $x / R=0.05$ above the surface of the disk, where $\mathrm{x}$ is the direction normal to the disk.

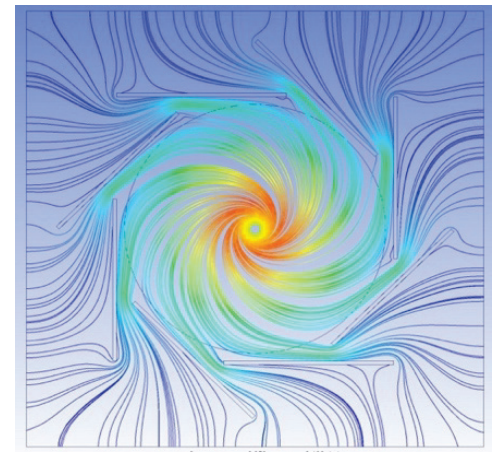

Fig. 6: Plan view of streamlines on a plane at $x / R=0.05$ above the surface of the disk. $R a_{D}=1.3 \times 10^{7}$.

Fig. 7 shows the surface heat flux of the disk for the model with vanes. The surface heat flux follows a similar swirling pattern as the flow over the disk, suggesting that the swirling flow is related to the heat flux from the disk.

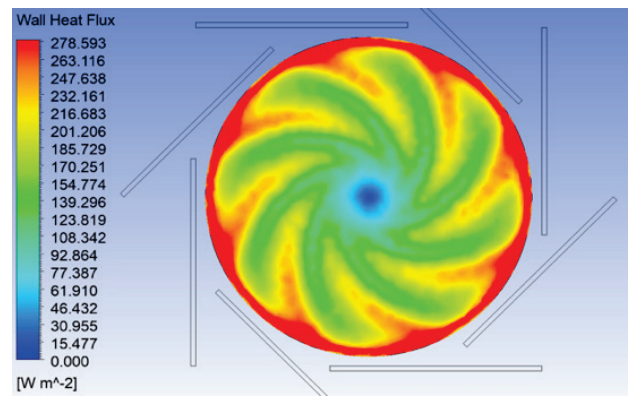

Fig. 7: Surface heat flux with swirl. The pattern of the surface heat flux follows that of the swirling flow. $R a_{D}=1.3 \times 10^{7}$.

\section{B. Secondary Flow and its Impact on Heat Transfer Rate in the Swirling Model}

An examination of the flow pattern on a plane approximately normal to the primary flow reveals regions of downwash and upwash that correspond to regions of high and low heat surface flux, respectively. Fig. 8 shows velocity vectors and streamlines of the secondary flow near the surface of the disk simultaneously with the surface heat flux. Fig. 8 shows that where the secondary flow impinges on the plate, there is high heat flux and where the secondary flow lifts off the plate, there is low heat flux. 

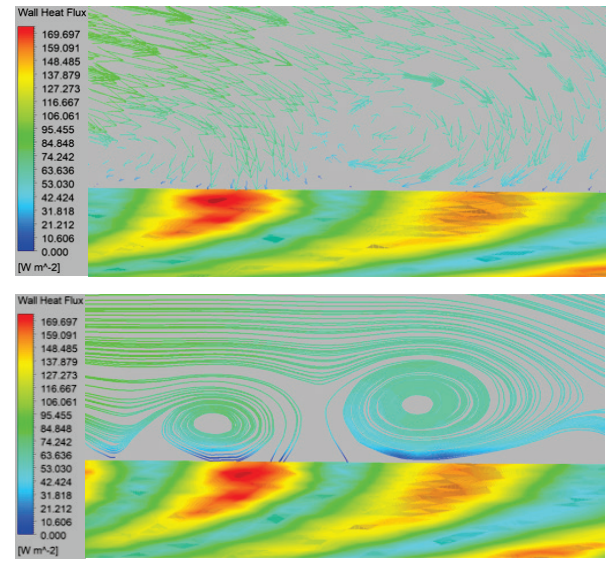

Fig. 8: The velocity vectors (top) and streamlines (bottom) above the disk on a plane approximately normal to the primary flow. The surface of the disk is coloured to show the surface heat flux. $R a_{D}=5.12 \times 10^{6}$.

Fig. 9 shows that the heat flux along a radial line is higher for the swirl model when compared to the model with no swirl. In the swirl model, the downwash from the secondary flow causes this increase in heat flux over some areas on the disk. The improved fluid mixing near the surface, as a result of the strongly swirling secondary flow, may be why the overall heat transfer rate is higher for the model with swirl compared to the model with no swirl.

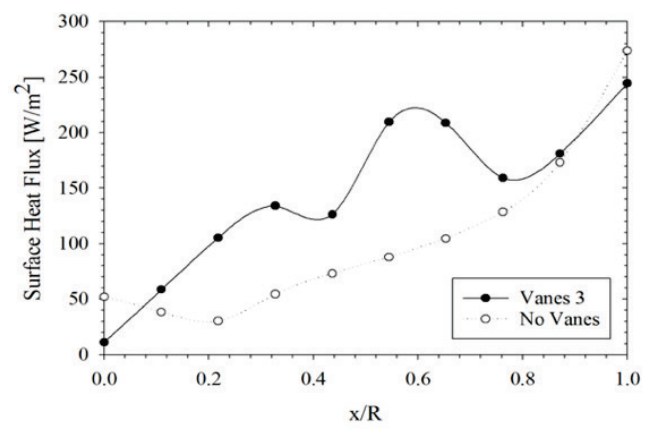

Fig. 9: The surface heat flux along radial lines, representative of the average heat flux for the swirl and no swirl models, plotted against $x / R$, the nondimensionalized distance along the line $\left(x / R=0\right.$ is the center and $x / R=1$ is the edge). $R a_{D}=1.3 \times 10^{7}$.

\section{CONCLUSION AND RECOMMENDATIONS}

The effect of a passively induced swirling flow over a heated horizontal circular disk under natural convection has been investigated numerically and experimentally. The following conclusions may be drawn from this study: 1) A passively induced swirling flow may increase the average heat transfer from a horizontal circular surface by up to $35 \%$, depending on the vane design and Rayleigh number. This is confirmed by the increase in heat transfer between the swirl and no swirl models measured numerically and experimentally. 2) The secondary flow in the model with swirl included regions of downwash and upwash that corresponded to areas of high and low heat surface heat flux, respectively. 3) More extensive design and iteration of the vane geometry may be needed to find the design which results in the highest heat transfer enhancement. 4) Further investigation is needed into the discrepancy between the numerical model of a disk under no imposed swirl and published literature of the same problem. 5) Experiments over a wider range of Rayleigh numbers are needed to further confirm the heat transfer increase from the swirling flow.

\section{ACKNOWLEDGMENT}

The authors would like to acknowledge Mr. Alan Machin at Ryerson University for his expertise in machining the experimental model and his advice on matters regarding MZI experiments. The support of the Undergraduate Research Opportunity (URO) at Ryerson University and NSERC are both gratefully acknowledged.

\section{REFERENCES}

[1] V. A. F. Costa and A. M. G. Lopes, "Improved radial heat sink for led lamp cooling," Appl. Therm. Eng., vol. 70, no. 1, pp. 131-138, 2014.

[2] M. Corcione, Natural convection heat transfer above heated horizontal surfaces, 5th WSEAS Int. Conf. on Heat and Mass transfer, Acapulco, Mexico, pp. 206-211, 2008.

[3] M. Al-Arabi and M. K. El-Riedy, "Natural convection heat transfer from isothermal horizontal plates of different shapes," International Journal of Heat and Mass Transfer, vol. 19, (12), pp. 1399-1404, 1976.

[4] W. W. Yousef, J. D. Tarasuk and W. J. McKeen, "Free Convection Heat Transfer From Upward-Facing Isothermal Horizontal Surfaces," Journal of Heat Transfer, vol. 104, (3), pp. 493-500, 1982.

[5] K. Kitamura and F. Kimura, "Fluid flow and heat transfer of natural convection over upward-facing, horizontal heated circular disks," Heat Transfer-Asian Research, vol. 37, (6), pp. 339-351, 2008.

[6] A. D. Machin, "An Experimental Study of Free Convective Heat Transfer from a Vertical Flat Plate in the Presence of Louvers.", ProQuest Dissertations Publishing, 1997.

[7] D. Naylor, "Recent developments in the measurement of convective heat transfer rates by laser interferometry," International Journal of Heat and Fluid Flow, vol. 24, (3), pp. 345-355, 2003.

[8] Naylor, D., "Laser Interferometry", Ryerson University Library and Archives Digital, Repository, http://digital.library.ryerson.ca, Technical Report \#HT-01-2017, 34 pages, April 2017.

[9] Goldstein, R.J., "Optical Measurement of Temperature", in Measurement Techniques in Heat Transfer, E.R.G. Eckert and R.J. Goldstein, Eds., Slough, England: Technivision Services, 1970, pp. 177-228.

[10] J. S. Slepicka and S. S. Cha, "Stabilized nonlinear regression for interferogram analysis," Applied Optics, vol. 34, (23), pp. 5039, 1995.

[11] M. E. Poulad, D. Naylor and P. H. Oosthuizen, "Measurement of TimeAveraged Turbulent Free Convection in a Tall Enclosure Using Interferometry," Journal of Heat Transfer, vol. 133, (4), pp. 042501, 2011.

[12] Touloukian, Y.S., Liley, P.E., Saxena, S.C., "Thermal Conductivity: Nonmetallic Liquids and Gases", in Thermophysical Properties of Matter, vol. 3, New York: Plenum Publishing Corporation, 1970.

[13] Touloukian, Y.S., Makita, T., "Specific Heat: Nonmetallic Liquids and Gases", in Thermophysical Properties of Matter, vol. 6, New York: Plenum Publishing Corporation, 1970.

[14] Touloukian, Y.S., Saxena, S.C., Hestermans, P., "Viscosity: Nonmetallic Liquids and Gases", in Thermophysical Properties of Matter, vol. 11, New York: Plenum Publishing Corporation, 1970. 